II.

Ueber einigo der Verbindungen des oxygenirtfaizfauren Gas und des Sauerftoffs mit andern

Körpern,

u nd

iiber die chemifchen Beziclungen, worin diefo Principe zu den verbrennlichen Körpern ftehen.

ron

Humpar Davx, Esq.

(Vorgelefen in der königl. Societât su London am 15. Nov. 1810.) ")

In meiner letzten Vorlefung habe ich die Ehre gehabt, der königl. Societät eine Reihe von Thatfachen mitzutheilen, welche mich geneigt máchten zu glauben, der Kürper, den wir in der neuern Chemie uneigentlich oxygenirt-falzfaures Gas nennen, fey bis jetzt noch nicht zerlegt worden, ley vielmehr für einen chemilch-einfachen, in mehrern feiner Eigenfchaften dem Sauerftoffgas ähnlichen Körper zu halten. Bei meiner heutigen Vorlefung habe ich zur Abficht, der königl. Societät im Detail die Verfuche mitzutheilen, welche ich ange-

*) Frei üborfetst aus dem Originale, nach oinem einzelnen Abdrucke aus den Philofophical Transactions of the Roy. Soc. of London. London 1811. 35 S. 8., welchen ich Hetrn Davy [elbit verdanke.

Gillert. 


\section{[ 44$]$}

fiellt habe. um die Natur, die Eigenfchaften und die Verbindungen dieles merkwürdigen Körpers, und die Verwandt chaften deffelben zu den verbrennlichen Körpern, Vergleichungsweife mit denen des Sauerftoffs,noch mehr.aufzuklären; und auf ihnen mögen einige allgemeine Anfichten und Schlïfe folgen, welche die chemifchen Kräfte verlchiedener Arten von Materie, und die Verhältniffe betreffen, in denen fich diefe Materien mit einander verbinden. Ich habe mich, feit der letzten Sitzung der Societät, falt anhaltend mit diefen Unterfuchungen belchäftigt; doch reichte diele Zeit nicht hin, irgend eine derfelben ganz zu vollenden. Ich fchmeichle mir indels, dafs bei Gegenftänden, die nicht nur für die höhern Regionen der chemifchen Phyfik, fondern auch für die ökonomilchen Anwendungen der Chemie, von fo grolser Wichtigkeit find, auch diefe noch unvollkommenen Arbeiten nicht unwillkommen feyn werden.

1) Ueber dio Ferbindungen des oxygenirt-falyfauren Gas und des Sauorfoffes mic den Metallen aus den fouerbofiändigon Alkalien.

Wie grols die Intenfität der Verwandt fchaft des Kaliums zu dem oxygenirt-falzfauren Gas ift, lälst fich daraus abnehmen, dafs das Kalium fich in diefem Gas von lelbft entziindet, und dann mit vieler Lebhaftigkeit verbrennt. Ich habe mich durch mehrere mithfame Verfuche überzeugt, dal's während-diefes Verbrennens kein Walfer abgelichieden 


\section{$\left[\begin{array}{ll}45 & \end{array}\right]$}

wird; dafs I Grain Kalium dabei ung̀efähr r, r Kub. Zoll oxygenirt-falzfaures Gas von mittlerem Druck und mittlerer Temperatur ver[chluckt; und dals beide Körper mit einander in eine neutrale Verbindung treten, welche fich nicht verändert, wenn man Gie fchmelat.

Bei den Verfuchen, aus denen ich diefe Schlüfre ziehe, lag das Kalium in einem Schälchen (tray) von Platin; es wurde in einem luftleeren Gefärae erhitzt, damit es zuror alles Waffer zerfetzen follte, welches die Kali-Rinde einfchluckt, die fich auf dem Kalium, während es mit der. Luft in Berührung ift, bildet, und das oxygenirt-falzfaure: Gas swurde zuror durch falzfauren Kalk von :allem.Wafferdampfe befreyt. Bedeutende Maffen von Kalium entzünden lich im oxygenirt-falzfauren Gas nicht, ohne darin erhitzt zu werden; und fo oft ích das Kalium auf dem Glafe der Retorte felbft fchmolz, zerfprang die Retorte; ja diel'es gelchah felbft zwejmahl, als ich das Platinfchälchen brauchte; mit folcher Heftigkeit geht das Verbrennen vor fich. Hat inan das oxygenirt-falzfaure Gas nicht zuvor von Wafferdampf befreyt, oder ift das Kalium unmittelbar zuvor mit der Luft in Berïhrung gewefen, fo fetzt lich in beiden Fällen während des Verbrentnens ein wenig Feuchtigkeit ab. Sind aber das Kalium und das oxygenirt-falzlaure Gas beide rein, To entfteht, (wie ich angegeben hatte,) blos eine Verbindung aus beiden, und zwar. diefelbe, welche man durch Glühen von lálzliuurem Kali erhält. 


\section{f 46$]$}

In Sauerftoffgas verbrennen Kalium und $\mathrm{Na}$ tronium mit weit geringerer Lebhaftigkeit, als im oxygenirt - falzfauren Gas. Diefe und einige andre Erfcheinungen lielsen mich vermuthen, dafs beide Metalle eine geringere. Verwandtfchaft zum Sauerftoff als zu dem oxygenirt - falzfauren Gas haben; und einige.Verluche; die ich angeftellt habe, bewiefen, dafs diefes in der-That der Fall ift. Doch ehe ich in das Detail derfelben eingehe, mufs ich die Natur der.Verbindungen des Kalium und des Natronium mit dem Sauerftoffe, und des Kali und Natron mit dem Waffer, vollftändiger unterfuchen, als es bis hierher gefchehen ift.

Ich habe in meiner letzten Baker'fchen Vorlefung angeführt, dafs Kalium und Natronium beim Verbrennen in Sauerfooffgas Kali und Natron in einem Zultande äufserfter Trockenheit, in welchem fie fehr fchwer zu l'chmelzen find, hervorbringen *). Ich bediente mich, wie ich angegeben habe, bei den Verfuchen, aus denen ich diefen Schlufs zog, der Schälchen aus Platin, und da ich fand, dafs diefes. Metall dabei. oxydirt wurde, erhitzte ich die Retorte ftark, um allen Sauerftoff, den das Platin verfchluckt hatte, wieder auszutreiben; wenn ich diefe Vorficht nicht brauchte, fand ich immer viel mehr Sauerftoffgas verlchluckt, als fich durch Erzeugung der beiden Alkalien erklären

7) Der Lefer fuclet diefe Baker'fche Vorlefung Davy's in Band 7 . der N. F. diefer Annalen, und die Stelle, welche Hr. Davy hier meint, N. F. B. 7. S.58 f. Gilbert. 


\section{[ $\quad 47$ ]}

liefs. Jedesmahl, wenn-ich Kalium oder Natronium in atmiofphärijfcher Lufc bei mälsigem Erwärmen verbrannt habe, fand ich, dafs' die erften Producte äufserft fchmelzbare, röthlich braune Körper waren, welche im Waffer ein lebhaftes Aufbraufen bewirkten, und bei ftarkem Erhitzen an der Luft, auf Platin, zu trocknen Alkalien wurden; Erfcheinungen, welche mich verleiteten, fie in den frühern Zeiten diefer Unterfuchungen für erfte Oxyde (prot-oxi-des) *) von Kalium und Natronium zu haiten. $\mathrm{Da}$ ich aber in der Folge fand, dafs.fie mit Eifenfeilen verbrennęn, und dafs lie Platin und Silber fchnell oxydiren, fo liels ich meine Meinung über fie unbeftimmt, bis ich ihre Natur genauer würde unterfucht haben.

Diefe Oxyde haben feitdem die Aufmerkfamkeit der Herren Gay-Luffac und Thenard auf fich gezogen, wie ich aus dem Moniteur 5. Juli 1810 **) erfehe, und diefe gefchickten Chemiker haben entdeckt, dals es Oxyde im Maximo (peroxydes) des Kaliums und des Natroniums find, von denen das erfte, nach ihnen, 3 mahl to viel Sauerftoff als das Kali, und das zweite $x$ mahl fo viel Sauerftoff als das Natron enthalten.

*) Ich überfetze diefen Atedruck hier nicht durch Oxydül, weil bei Körpern, die mehr als zwei Oxyde haben, dadurch Zweideutigkeit entftehen würde. Das nach dem Oxydül folgende Oxyd müfste dann das exfte Oxyd u. f. f. genannt werden, wodturch man mit der $T$ b o m Co $^{\prime}$ 'fchen von $D$ avy angenommenen Bezeichnungsart der Oxyde it Witlerfpruch kommen würde.

$G \| l b=t$.

") Uoberfetar in Band 6. diefer Annalen S.285. Gilbert. 


\section{[ $\quad 48$ ]}

Diefe intereffauten Refultate haben fich mir im Allgemeinen beftätigt, obgleich ich kein Mittel habe auffinden können, den Sauerfoffgehalt diefer neuen Oxyde genau zu beftimmen. Werden fie auf einem Metall gebildet, fo geht ftets eine ftarke Oxydirung diefes Metalls vor fich, felbft des Ptatins. Als ich das. Platinfchälchen mit ge[chmolzenem falzfaurem KaIi überzogen hatte, verfchluckten in einem Verfuche 2 Grains Kalium 2,6 Kubikzoll Sauerftoffgas, dagegen in einem andern Verfuche I Grain Natronium 1,63 Kubikzoll-Sauerftoffgas; und doch bin ich geneigt zu glauben, dafs zugleich mit den Oxyden im Maximo auch etwas Alkali ${ }^{\star}$ ) entftanden $f_{e y}$. Das Barometer ftand während diefer Verfuche auf 30,12 Zoll, das Thermometer auf $62^{\circ} \mathrm{F}$. In dem zweiten Verfuche hatte die Schneide des Platinfchälchens auf das Natronium eingewirkt und war oxydirt ${ }^{\star}$ ). Die Farbe diefer auf falz gebildeten Oxyde im Maximo war bei den aus dem Kalium glänzend Orange, und bei dem aus dem Natronium, dunkles Orange. Mit Wafler oder mit Säuren gaben fie Sauerftoff her, und beim Erhitzen

*) Das heifst, Kali- und Natron-Hydrat, welche die Oxyde im Minimo enthalten.

Gilberc.

**) Nach den Herren Gay-Lu $\iint_{a} c$ und Thenard verfchlucken Kali und Baryt Sauerftoff beim Erhitzen. Die Einwirkung der fouerbeftändigen Alkalien und des Baryts auf Platirl febeint mir daher auf der Bildung diefer ihrer Oxyde im Maximo zu beruhen $;$ l eine Demerkung, die ohne Zweifel diefe gefelickten Chemiker in der umftindlicheren Erzäblung ihrer Verfiche fchon werden gemacht haben.

Davy. 


\section{[ 49 I}

mit einem metallifchen oder brennbaren Körper verwandelten fie fich in Alkali, wie die Herren $G$ a y-

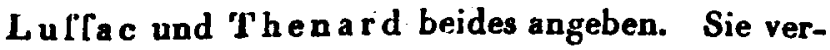
dicken die fixen Oehle, und bilden eine Verbindung mit ihren, welche Curcume-Papier ohne Hinzufügen von Waffer nicht rüthet.

Bringt man in Röhren aus reinem Glafe Kalium mitgefchmolznem Salpeter in Berührung, fo entfieht blos ein leichtes Funkenwerfen (Scintillation), und der Salpeter wird rothbraun. Es entlteht dabei Stickgas, und es bildet lich das Kalium - Oxyd im Maximo. Ich hoffte, wenn ich das fich entbindende Stirkfas und das Sauerftoffgas, welches aus dem entftandenen Kalium-Oxyd darch Waffer entwickelt wird, mälse, würde ich das MilchungssVerhältnifs dipfes Oxyds im Maximo genau autlinden können. Aber I Grain Kalium entbindet hierbei nur $\frac{1}{10}$ Kubikzoll Stickgas, und das rothe Oxyd [das Kalium-Oxyd im Maximo] beim Einwirken auf Waffer weniger als $\frac{5}{2}$ Kubikzoll Sauerftoffgas, daher wahrfcheinlich in diefer Operation Kali zugleich mit dem Oxyd im Maximo gebildet wird. Bringt man mit gefchmolznem Salpeter Natronium in Herührung, fo entfteht ein heftiges Verbrennen. In zwei Verfuchen mit I Grain Metall zerfprangen die Gläsröhren mit der Heftigkeit einer Explofion. Mit $\frac{x}{2}$ Grain Natronium glückte es mir, die feften Producte des Verbrenuens zu erhalten; ${ }^{\bullet}$ aber es hatte fich kein Peroryd gebildet, denn aus der Moffe entwickelte fich im Waffer kein Sanerftoffgas.

Aonal. d. Phylik. B. 39. St. x. J. 181, St, 9. 


\section{[ 5o ]}

Wird Kalium in einer Ketorte aus reinem Glale verbrennc, lo ilt das Product zum Theil Kali, zum Theil Peroxyd, und bei langem Rothglühen wird das Peroxyd ganz zerfetzt. - Als ich I Grain Kalium allmählig in Sauer/toffas in einer kleinen. grünen Glasretorte erhitzte, verbrannte es langfam und mit einer fchwachen Flamme, und es wurden to Kubikzoll Sauerfoffgas verfchluckt; ich brachte darauf die Retorte zum heftigen Gluhen, und es wurden to Kubikzoll Sauerftoffgas wieder ausgetrieben; Barometerftand 30,1 Zoll, Thermometerftand $63^{\circ} \mathrm{F}$.

Ninımt man die electrifche Zerfetzung des Kali und des Natron mit einer Volta'Tchen Batterie von 500 bis 1000 Plattenpaaren in roller Wirkfamkeit vor, fo verbrennen die Metalle in dem Augenblicke, weno fie entflehn, und bilden die Peroxyde; und aus Ritter's Verfuchen wird es wahrfcheinlich, dals diefe Körper ") gleichfalls in Kali in der Rette des Volta'fchen Apparats an der pofitiven Oberfiiche entftehen können. - In meinen frïhern Verfuchen über das Kalium und Natronium hielt ich die \{chmelzbaren Subftanzen, welche in dem Volta'Tchen Kreife in den beiden Alkalien an der negativen Oberflïche entftehen, fo gut wie die, welche fich bilden, wenn die Metalle der Alkalien der Wärme und der Luft ausgefetzt werden, für Oxydiile (prot-oxydes), und für ähnlich den Pro-

') d. b. die Peroxyde orler Oxyde im Maximo. Cillars. 


\section{[ 51$]$}

ducten, die beim Erhitzen der Alkali - Metalle mit geringen Mengen Alkalien entflchen. Diele letzten Operstionen, in denen, wie ich glaubte. Oxydiule oniflünden, habe ich wiederholt. Werden Kalium oder Natronium 'mit der Hilfte ihres Gewichts on glïhend gefchmolzoem Kali oder Natron in Glasrühren erhitzt, lo verwandılt lich zuerft ibre Farbe in glünzend Lafurblau, dann entwickelt fich eine Menge Walterltoffgas, und zuletzt bildet lich eine graue, cohärente, in Rarker Rothgfühehitze nicht Ichmelzbare Malle, welche beim Einwirken von

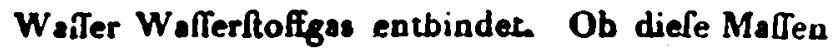
wabro Oxydülo (erfto Oxyde) lind, oder blalso Meogungen der Alkati-Metalle mit den Alkalien, (vielleicht mit Silicum aus dem Giafe vermifcht,) unternehme ich jetat nicht zu ont fchoiden. - Kalium, das auf diefelbe Art mit befchmolznem Kali in einer Platinröhre erhitzt wurde, gab, nachderm es fich entzündet hatte, eint dunkle Maffe, die mit Waffer aufbraufte; aber lellsf in diefem Fall fcheint eine Legirung aus Kalium und Platin mit in das Spiel zu kommen, und die Malle kein Oxycluil, fondern blos trocknes Alkali, mit diefer Legirung vernengt, zu leyn.

Da wir die reinen Alkalien erlt leit der Entdeckung:des Kalium und des Natronium kennen gelernt baben *), und ihre Eigenfchafien noch nir.

D 2

-) Stabl kam der Entdechung Jor roinen Alkalien Tohr nabe. Er cementrirte fefteo kauftilchee Kali mit Eifon. 


\section{$\left[\begin{array}{ll}5 \mathrm{a} & ]\end{array}\right.$}

gends befchrieben find, fo will ich fie hier in der Kürze ongeben: Verbrennt man Kalium oder $\mathrm{Na}$ tronium auf Platin in Sauerfoffgas, und erhitzt lie bis zum Rothglïhen, um die Peroxyde zu zerfetzen, fo erhält man Alkalien von gräulich grüner Farbe. Sie find härter als gewöhnliches Kali oder Natron, auch [pecifilch fchwerer, lo weit fich darüber nach einem unvollkommnen Verfuche urtheilen läft. Sie werden erf in Rarker Rothgluihehitze vollkommen Aiülig, und rerdampten langfam, wenn die Hitze noch höher getrieben wird. Werden kleine Mengen Waffer mit ihnen in Bęrührung gebracht, fo erhitzen fie lish heftig, werden weifs, und verwandeln fich in Hydrace, und dann find lie leicht lichmelzbar und Aüchtig. - - Werden Kalium oder Natronium auf Metallfreies Glas verbrannt und dann ftark erhitzt, oder wenn man durch Einwirkung ron ein wenig Wafler aus diefen Metallen Kali oder Natron bildet, fo nähert lich ihre Farbe

feile in lang forgeferster Hitse, and fogte, dofo men auf diefo Art oin Rali orbalto, wolchee ..ger fohr (valde) kau. Itifcb" Sey (Spectin. Bech. P. 2. p. 255.). Auch bildete er kouftifcbos Alkali durch Zerfetrung ron Salpeter durch Metalle (daf. p. 253.). Ich Gode, dafo, woon men Salpe. ter in oinem Platintiegel durch boftiges Rotbglübn serlecsi, eind gelbe Subftens ourückbleibr, die aus Kali ond gelbew Platinoxyde, dem Anlebeine aach miteinander chemifch verbunden, beftebt. Das Koli, welcbes in dem Flin. tenlaufo, worin men Kalium auf die bekennte Art bildet, zugleicb mit diefem Metalle ubergebt, bat eino Orangefarbe, und lotat, weno man es in IT aller sulöh, Eilenoxyd ab. Wabrfcheinlich hat reines Kali Verwandtifbaft su mebreren Metalloxydeo.

Davy. 


\section{[ 53$]$}

dem Weifs; in andern trabrnebmbaren Eigenlichaften gleichen fie aber denen durch. Verbrennen auf Metalle erhaltenen Alkalien, und baterfcheiden fich durch ihre fchwero Sobmeltbarkeit auf tine ausgozeichnete Art ron dem durch Alkabol bereiteten Kali und Natran.

Hr. d'Arcet, und noch befimmter. Hr. Berthollet, haben gofchloffea; dafs der Gowjchtsverluft, der fich ergiebt;. wenn gewöhaliches, ge: fchmolznes Reli oder. Natron mit Säuren verbunden werden, ron dem Waffer diefer Alkelien herrübrt, wedches nicht in diefe Verbindung mit eingeht; und Hr. Bertbollet hat das Waffor im Kali aúf 13,9 , Hr. d'Arcet auf 27 bis 28 Procent, und letzterer das Waffer in Natron auf 28 bis 29 Procent befirmmt '). Ich habe in peiner letutern Baker'fchen Vorlefung angefiibrt, dafs meine Verfuche mich auf das Refultat gefuihrt haben, dafs gefchmol: zenes Kali in 100 Theilen ungefähr 16 bis 17 Theire Wialfer enthalte, verglichen wit dem durch Verbreonen voa Kalium in Sauerfoffgas gebildeten Kali "*). Ich 20 g diefen Schlufs ous Verfuchen mit Kiefelerde und Kali, die ich miteinander fchmolz, wohei ich snnahm, dals der Gewichurerluft der Menge Wafter in dem Kali gleich leg. Dals je Waffer wirklich aus dem gefchmolsnen Kali oder Natron wure aufgefangen worden, ift mir nicht bekunnt, und doch Tchien mir diefe Darftellung dés

') Diele Annalen, Nexe Folge, B. 2. S. 4n. Gllbere.

"•, Diefo Annalon, Nowe Folge, B. 7. 8.62. Anm. Gllbort. 


\section{$\left[\begin{array}{lll}54 & \end{array}\right]$}

Walters zho völtigén Aufklärung des Gegenftandes nöthig su Seji.

Ich orhitzte daher in einèr. Retorte aus grünem Glafe 40 Grain Kali, das eiuige Minuten lang im Glühen gevireten war, 'rmit: yoo Groin Boraxfâure, welclse ich faft eine Stunde lang im:Weilsglühn erbalten batrei- Dio-Heturto !rar Jorgfältig gewogen worden, vand wurde' mit einérn kleinén, gleichfalls gewogenen Récipienten verbunden.' Darauf wurde die Kuget der' Retorte allmählig erhitzt, bis fie kirfrhroth glühte. In ver Retorte zeigte lich ein heftiog Aufbrarren, und in Halle derlelben condenfirté fich eine Flüligkeit, welche in die Vorlage überging. Als der Prorels vollendet war, wurde. die ganze Ketorte fark erhitzt. Es fand lich, dals Gie $6 \frac{1}{3}$ Grain an Gewicht verloren, und dafs der Recipient 5,8 Grain an Gewicht zugenonmen hatte. Die Flüligkeit, welche er enthielt, war Waffer, worin fich eine geringe Menge Borax[aure aufgelül hatte, und welches. nach dem Abdampfen keinen walirnehmbaren Rückftand liefs.

Ein äholicher Verfuch, den ich mit Natron, das bis zum. Rothglühen erbitzt worden war, annellte, in welchem aber das iibergegangene Waffer nicht gewogen wurde, zeigte in 100 Theilen Natron 22,g Theile $\mathrm{W}^{\prime}$ affer.

Man köunte hier das Bedenken haben, ob nicht ein Theit des Waffers, das in diefem Proceffe entbunden wurde, aus der Boraxfaure berrühre, oder durch Einwirkung derfelben auf die Alkalien 


\section{[ 555 ]}

gebildet worden iey? Der folgende Verfuch beveilt aber, dals diefes in keiner wahrzunehmenden Menge gefchehn [eyn konnte. Ich erhitate bis zum Hothglühen 8 Grain Kalium mit 50 Grain Boraxfäure in einem Platinrohre, das mit einer Glosröhre, die fehr kalt erhalten wurde, verbunden war; in diefem Proceffe entband lich aber gar keine Feuchtigkeit. Ich vermilchte wenige Grain Kalium mit rothem Queckfilber - Oxyd, und entzïndete die Mengung in Berührung mit Boraxfaure; es erfchien ober koin andres olaftifch-Aüliges Product als OueckGilberdampf: Darauf vermifchte ich reines, aus Kalium gebildetes Kali mit trockner Boraxfäure und erhitzte fie bis zum Rothglühen; es entftand baffches' boraxfaures Kali, zeigte fich aber keine Spur von Feuchtigkeit *).

-) Man darf indefo are diefen Verfuchen nicht [chliefsen, dale Boraxfauro, die bis sum Weifoglühen erhiter worden, gans Wallerfrey Loy; fie bewoilen blof, dafo eine fólche Säure beim Vorbinden mit roinem Kali koin Waller in der Rouhglühtikse hergiebt. Ich babe gafunden, dafe Boraxfaure, die vollkommen im Flulle, und larige vor dem Gobläe einer ETo gewefen ift, und die linget aufubraulen aufgehört hat. Bläschen Walferftoffges hergiebt, wenn man trockne Eilenfoile auf fie oinwirken lafac. Ich that in einen Platintiegel su 54 Grains Boraxfiure, welche in rollkommenem Fluffe waren, 75 Graine Flinegles, das suvor bis zum Woileglühn erbitzt und dann nemirtolbar in einem beifsen eilemen Mörfer su einem Pulver serltofen worden war; als die Hitse fo erböbt wirdo, dafs beide in Veobindung traten, enuftend viel Aufbraulen, ond aach beftigem Glühen während einer halben Stuade fand fich ein Gewichuverluft von $3_{\xi}^{2}$ Grain. - Ich Gnde, dafo die bis zum Rothglühn erbitsten Verbindungen von Boraxfäure mit Kali 


\section{[ 56. ]}

Es erhellet aus diefer Reihe von Thatlachen, dafs das gowöhnliche, Kali und das gewühnliche $\mathrm{Nam}$ tron Hydraco find; die durch Verbrennen der Alkali - Metalle dargeftellten Körper dagegen find dio reinen Metall-Oxyde, frey yon Walfer, (fo weit wenigltens unfre Kenntnifs reicht.) Nach dem Gewichtsverluft zu urtheilen, den:das gewöholiche Kali und Natron leiden, rwenn lie mit Boraxfaure verbunden'werden, fcheint der Waffergehalt des er-

öder mit Natron an Gowicht verlieren, weun fie su einor viel böberon Temperatur gebracht werden. In dem Laboratorio meinos Freundee, Jobn Goorge Childern Esq., vermifchten wir a. B. 71 Grain Kali-Hydrat mit 96 Grain in eínem Gebläofen möglichft ftark erhitater BoraxCaure, wahread foe mit einander in Mochglühbises gefehmolsen murden, vorloren fo "I Grain, ale abor dio Hirze bis sum Weifsglüinn erböht wurde, Ttieg der Gewichtsvertuft auf 3 Grain. In einem ahnlichen Verfuche verloren 55,5 Grain Natron-Hydrat und 80 Grain Boraxfiure; wahrend eittor halben Stunde, wo fio von Zeit su Zeit gemogen.wurden, immerfort an Gewicbt, obgleich die Hitso hiufig bis sum. Weifglübn ftieg, und ihr ganzer Ge. wichtororlult betrug if Grains, moron weoightens it Grain auf die 8quues su kommen fcheinen. Es verloren 95 Grain Natron und 140 Grain trockngs Flintgles, dis mit einander in oinem Platintiogol weils gegliiht wurdon, 22,2 Grain an.Gewiobt; ich that aufs neue 80 Graia Boraxglas binsu, und es erfolgte aufiene Aufbraufen, und nach wanig Minzten heftigem Glühen hatte der Gewichteverluft um $4 \frac{1}{2}$ Grain sugenommen. Mit welcher Kraft das Walter in anders Fälten an gewiffen Körpem adharirt, seigt der Verfuch, den Hr. Berthollet im aten Bande der Mim. d'Aremell p. 47. [or betrifft den Baryt] erzählt. Eo lalot fich in dor That nicht behaupten. dals irgend eine neutrale Verbindung oder eine fefto Saure je gans wafterfrey foy; nur die orfton Antboilo Waller laften fich loicht ab. Ccheiden

Davy. 


\section{$\left[\begin{array}{lll} & 57 & \end{array}\right]$}

fiern 19 bis 20 , und der des letztern 23 bis 25 Procent 24 betragen.

Dals aber Kalium und Natroniua nicht etwa Hydrure von Kali und Natron find, das fcheint fiir die hellfehenden chemifchen Naturforlcher in Britannien, die mit den Fortlchritten der Wiffenfrhaft Sohritt gehalten haben, keines weitern Beweiles zu bedürten, befonders da die finnreichen Vertheidiger der eben eqwähnten Moinung, die Herren Gay-Luflac und Thenard, in der angeführten Notiz im Moniteur, bekannt haben, dals diele Meinung unhaltbar ley *).

-) Bei einem Gogenftordo, der in lo geasuer Terbiadung mit der ganzen Aaficht der Chemie, und mit lo viel neven Unterfucbungen fteht, kann es jedoch nicht lchaden, der Beweifo durch Thatheben noch mobrere su fuhren. Herr Dalton im 2. Bande foines New Syftem of Chemical Phllofophy, wolchon or mir suxufchicken dio Güte gebabt hat, [p̈ticht auf den orften Seiten von Keli und Natron als ron Oxyden, auf den letzten Seiten aber find fie ibm chemiçch einfacho Körper, und die aus ihnen gebildeten Netalle Verbindungen ron Kali oder Natron mit WallerItoff. Er giebt keine einzige Thatache für diofe Verāndrugg leinor Moinung an; feinen Hauptgrund nimmt er von dem Procello hor, durch den ich clas Kalium suerfi erhalten habo. Gowöholiches Kali ift ein Hrdraic; mittelf der Volu'Tchen Elecuricität erbält man daraus an der pofitiven Oberfäche Sauerltoff, nod en der negativen Ka. lium dor Saueritoff kömme aus den Wafler, und der Wafferftoff dee Walfers, meint Hr. Dalton, verbindet fich mit dem Kali su Kalium. Durch eine folche Scblufsfolgo liefso fich indefo ebenfalls beweifen, dalo Bley oder Kupfer Hydrure ibrer Oxyde find; denn wenn diefe Me. talle in dem Volta'chen Kreile auo ihren wäferrigen Auflofungen regulinifch sbgefchieden werden, entfteht 


\section{[ $\quad 58$ ]}

Ich komme nun zu den Verfuchen, welche ich itber die Gröfse derVerwandtfchaft des oxygenirtfalzfauren Gas und des Saueiftoffs zu den Mecallen der feuerbeftändigen Alkalien angeftellt habe.

Ich verbrannte I Grän Kalium in einer mit einem Hahne verfehenen Retorte aus grünem Glafe in

gleichfallo an der politiven Obertäche Sauerftoff, an der negativen Oberfächo aber kein Walfor. - Bei meinen erften Verfuchen über das Kalium und Natronium wendete ich nur cino geringo electrifche Kraft an, und da in ibnen nur lehr geringe Mengen der Metallo gobildet wurden, nahm ich kein Aufbraufen wahr. Nimme man aber 500 bis 1000 Plattenpanso zur Kalium - Bildung. โo fandet wäh. rend der Bildung der Metalle ein heftiges Aufbraúfen Statt, und Erzeugung eines zuwreilen mit Kalium verbundenen Walferftoffas (pocafjuretted hydrogeno). - Bringt man Kalium mit rotbglühendem Kali.Hydrat in Berührung, fo entbindet fich viel Wafferftofgas, and des Ganze verwandely fich in das Schwer Selumelsbare Kali. - Ich liefs in oinem Flintenlaufo 327 Grains Kali-Hydrat, die zuvor glüband gemacht waren, auf 745 Grtin Eifonfeilo oinnirketh, wolche bis sum Weilsglühen erbitst waren; etwas Wafferfoffges ging verloren, und etwas Kali- Hydrat blieb unzerfetzt; dennoch fing ich 235 Kubikz. breanbares Gas auf, und es bildeten fich 50 Grain Kalium und in grofser Menge eine Legierung aus Kalium und Eifen, fo dafo lich kaum sweifeln lälst, dafs allor aus dom, aerferzten Kali-Hydrat berrorgebrachto Walferftoff frei getrorden fey.

Herr D a l t o n glaube einige Aebnlichkeit mifchen Kalium und Natronium und swifehen den Verbindungen deo Wraferftoffe mit Schwefel, Phosphor und Arfenik, su Teba; ich woifs indefo nichts Aehnliches au finden swilchen Schwefel - Wasterftoff, einem Gas, das aufiuslich in Waffes ift, und die Eigenfchaften einer Säure hat, und einein börhft verbreanlichen feften Metall; das beim Verbrenzen Alkali orseugr. Eben lo gut liefse fich Kalium 


\section{$\left[\begin{array}{ll}59 & ]\end{array}\right.$}

Sauerftoffgas, und erhitate das entfandne Oxyd bis zum Rothglühen, um os in Kali zu verwandeln; es ivar \& Kubikzull Saueritoffges / verfchluckt worden. Darauf wurde die Retorte luftleer gepumpt und fehr reines orygenirt - falzfaures Gas hineingelallen. Augenblictlich nahm das Kali eine weifse Farbe an, und durch märsiges Erhitzen verwandelte lich das Ganze in falzfaures Kali; dabei wurden

mit Kobleofaure rergleichea. H. Dalto a fieht die Flüchtigkeit des Ralium und Natronium alo etwas an, das die Moinung, dafo fo Wallerfoff enibalien, bogünftige: aber fo find minder Düchug als Spiefglans, Arfonik und Tellurium, und fohr viel weniger als Queckfalber. Endlich beruft er lich auf ibs geringes fpocifilches Gewicht. Dielen suerf von Horsn Ritcer enforubrten Grund babo ich ill moinon vorigen Abbendlungon hiplinglicb geprüft; hier fubre ich our noch an, dals, wern Kalium eine Verbindung ron Walforftot mit Keliwire, Kali-Hydrat eben to viel Wafforftoff, dern Gowichw nacb, enchalien müfole, und überdiels noch ein zweites, leichtes, gesformiges Element, nomlich Sauerftof, von dem man eber eive Vorminderung als eine Vermehrung don fpecifichon Gowichce erwarten tollte. - H. Dal to a irri Gich, woon er p. 488. enoirirot, defo Kalium tuochnee Kali.Hydrat bilde, weno es Solpetergas odes oxydirtes Stickga serletse. Ich finde durcb fohr forgfalige Verluche, dafo Kalium diefon Kurpern den Saverftoff und etwas von ihsem Scickftoff entziebt, und eine fchmelabare Verbindung bervorbringt, dio Gch in der Rnthglüblicse. wäbrend lich Stickftoff und der uberfcbüfrge Saverfloff aus ibm entbinder, serfetst, und zu Kalı wird, eber vicht zu trocknem Kali-Hydrato. - Die HH. Gay.Luffa: und Thenard babea fich devon, hafs Kelium und Netronium piche Hydrure ron Kali uad Natron lind, dureb eine ahnlicbo Metbode mit der, die ich einige Monate zuvor gebraucht und bekennt gemacbe irabe. überseugr. neanlich dadurch, dafs fie neutrale Salse sus ihnen bildotea.

Davy. 


\section{$\left[\begin{array}{ll}60 & ]\end{array}\right.$}

1t Kubikzoll oxygenirt-Lalzfaures Gas verfchluckt, und gema \& Kubikzoll Sauerftoffgas erzeugt. Währead der ganzen Oporation ftand das Barometer auf 30,3 Zoll und das Thermometer auf $82^{\circ} \mathrm{F}$. Ich habe mehrere ähnliche. Verfuche angeftellt, aber dieles if der Einzige völlig zurerlälige. Nahm ich grǘsere Mengen Kalium, fo zerfprang die Retorte gewöhnlich während des Erkaltens, und brauchte ich metallne Schälchen, fo war es niche möglich, zu pinem genauen Kefultate zu kommen. Das Kalium war zu einer dünnen Platte ausgedehnt worden, und oxydirte fich daher fehr, bevor es in die Retorte kam, weshalb es etwas weniger Sauerftoffgas verfchluckte, als obnediefs der Fall gewefen leyn wïrde. Um das Waffer in der entftandenen Kalikrufte zu zerletzen, wurde das Kalium vor dem Verbrennen im luftleeren Raume erhitzt; denn wenn ich diefe Vorficht nicht brauchte, fublimirto fich Kali-Hydrat und überzog den obern Theil der Pietorte, und daraus [chied das oxygenirt-\{alz aauro fias fowohl Woffer als Sauerftoffgas ab.

Dafs das oxygenirt falzfaure Gas aus dem Kali-Hydrate Waffer ablcheidet, zeigteslich glücklicher Weife in einem diefer Veriuche, in welchem ich diefes Gas zu Kalium - Oxyd im Maximaun fteigen ließs, welches in einer weiten Ketorte gebildet war. Das Kalium hatte fich in ihr mit eicer anliolindichen Krufte Kali - Hydrat überzogen, und während des Verbrennens war das Hydrat als ein weifses Sublimat in Sauerftoffgas aufgettiegen, das 


\section{[ $6 \mathbf{r}]$}

fich an dem obern Theile im Halfe der Retorte angefetzt hatte und vollkommen undurchlichtig war. Kaum war indefs das oxygenirt-lalzfaure Gas hinein gelaften worden, fo wurde diefes Sublimat fogleich durchfichtig durch Entwickelung ron Wafter, und als ich das Glas, da wo das Sublimat daffelbe beriihrte, éthitzte, wurde das Sublimat wieder undurchlichtig und Wafler davon getrieben (and wacer driven off).

In mehrern Fällen, in welchen ich reines oder mit Peroxyd vernulchtes trocknes Kali in oxygenirt-folzfaurem Gas erhitzte, fchied fich keine Feuchtigkeit ab, sulser wenn das Gas Walferdampf enthielt, und die Menge des Sauerftoffgas, welche in diefiem Proceîle bei ftarker Erhöhung der Hitze entwickelt wurde, ftimmte genau mit der ibèrein, die das Kalium verfchluckt hatte.

Als ich falzfaures Gas zu Kali, das durch Verbrennen von Kalium gebildet worden war, fteigen liefs, wurde in Augenblicke Waffer und oxygenirt-falzfaures Kalium (gewöholich falzfaures Kali genannt) gebildet. Ueber die Menge des Calzfauren Gas, welche das Kali zerfetzt, habe ich keinen genauen Verluch gemacht, wohl aber die Zerfetzung diefes Gas durch das Kali- Hydrat ein klein wenig unterfucht. Ich erhitzte in einem forgfältig gewogenen Platinfchälchen 10 Grain Kali-Hydrat zuın Rothglühen, brachte dann beide in eine Retorte, welche Luftleer gepumpt und darauf mit lalzlaurem Gas gefiillt wurde, und erhitzte das Kali-Hudrat 


\section{$\left[\begin{array}{lll}E_{2} & ]\end{array}\right.$}

mit einer Weingeift - Lempe; augenblicklich fchied Gich Waffer in grofser Menge ab, und es bildete fich falzfaures Kali. Darauf wurde ftarke Hitze gegeben, bis der Procels zu Ende war, und nun das Schälchen heraus genommen und gewogen; es hatte 218 Grain an Gewricht zugenommeri. Ich that ein klein wenig tropfbare Salzliure hinzu, um das Kuli vollkommen zu neutralifiren, und glühte darauf das Schälchen; das Gewicht hatte fich aber nicht verändert.

In den wenigen Verfuchen, welche ich über die Einwirkung des Natronium und des Natron auf oxygenirt-falzfaures Gas gemacht habe, waren die Erlcheinungen den hier erzählten völlig analog, nur dals das Natronium, wie zu erwarten war, faft die doppelte Menge von oxygenirt-falzlaurem Gas als das Kalium verfchluckte.

Wird gegliultes Kochfals mit Kalium erhitzt, fo zerfetzen lie fich fogleich, und bringt man die Milchung zum Rothglühn, lo erhält man reines Natronium. Ein Lehr bequemes Mittel, diefes Metall lich zu verfchaffen. Auch habe ich mir in den letztern Zeiten das Natronium nur auf diefem Wege bereitet. Bei dieler Operation entbindet fich kein Wafferftoffgas, und ich finde, dals 2 Theile Kalium mehr als I Theil Natronium hervorbringen.

Is erhellet aus der Reihe von Proportionen, welche ich in meinew letzten Auffatze *) mitgetheilt

") Oben S. 23.

Gilbert. 


\section{$\left[\begin{array}{lll}6 & 6\end{array}\right]$}

habe, dafs a Grain Kalium 1,08 Kubikzoll oxygenirt-falzfaures Gas verfchlucken, und dafs das aus \& Grain Kalium gebildete Kali ungefähr 2,16 Kubikzoll falzfaures Gas zerfezten muls; und diele Schätzungen Itimmen mit den Refultaten der Verfuche fehr nahe überein.

Das Nacron befteht, zu Folge der Schätzung, welche irh aus den Verfuchen in meiner letzten Baker'chen Vorlefung ") gefolgert habe, in too Theilen aus 25,1 Th. Sauerftoff und 74,6 Th. Metall, und diefem gemäfs wird das Verhältnifs (proportion), worin Natronium fich mit den Körpern verbindet, durch die Zahl 22 dargeftellt "*). Daraus ergiebt fich, dafs \& Grain Natronium nahe a Kubikzoll oxygenirt-folzlaures Gas verfchlucken, und in Natron verwandelt, nahe 4 Kubikzoll falzfaures Gas zerfetzen muls. Salzfaures Natron mülate nach dieler Anficht i Proportion von 22 Natronium und s

-) Diefe Annalen N. F. Band 7. S. 34. F, Gilbert.

") Oder durch die Zahl 44, wenn man Natron für das zweite Oxyd des Nocroniums nimmt, wie das aus den $S$. 47. f. angeführten Verfuchen wahrfcheinlich wird. Und unter diefer Vorauafetsung mufs man die Natron.Salso alo doppelte Proporionen von Säuro enthaitend fich deaken. In beiden Fällen aber muls die Proportion des Sauerfoffs 7,5 und des WalTerftofs i genommen werden, wenn fich gleich andre eben to gut alo diefo mit der allgemeinen Lehre von beftimmten Proportionen übereinftimmendo Zablen, als Diviforen oder Multipla follten finden laffen.

Davy.

[Den Vorfolg diefer Anmerkung findet man S. 34., wobin ich ibn sum beffern Verftündnits verfeust babe; dort auch das nütbige Licht su diefea Berechnungen. Gill e ert.] 


\section{[ 64 ]}

Proportion von oxygenirt - [alzfaurem Gas 3a,9 entlalten; eine Schätzung, die dem fehr nahe kümmt, wás Gch aus Dr. Marcet's Aaslyfe diefes Salzes folgern lälst Kali-Hydrat miilste beftehn aus I Proportion [Theilchen] Kali, das durch 48, und s Vafler, das durch 8,5 dargeftellt würde; und diefes gübé die Mifchung defrelben in 100 Theilen, zu 15,1 Th. Waffer und 84,g Th. Kali. Dagegen mürste Nasron-Hydrat beftehn aus I Proportion Natron zu 29,5 und a Wafler zu 8,.i, welches ouf 100 Theilo $22,4 \mathrm{Th}$. Waffer geben würde. Die vorhin angeführten Verfuche Itimmen nit diefen Schliifen fo genau überein, als fich"nur immer erwarten liefs. Auch die Mengen ron Kali und Natron, welche zu Folge dieler Schätzungen in verfchiednen neutralen Verbiadungen enthalten leyn müfen, kommen, wis man linden wird, fehr nahe mit den Mengen überein, welche die genauften Analyfen, befonders die des Hra. Berthollet, geben, und einige Abweichungen lind leicht zu erkliaren.

Ich habe in der vorhergehenden Abliandlung es wahricheinlich gemacht, dars das überoxygenirtfal:faure Kali eine Tripel-Verbinduug aus Kalium, oxygenirter Salzfiure und Sauerftoff ift. Diele Meinung wird durch die neuen Thatfachen beltatigt, welche wir hier über das Pernxyll des Kaliums haben kennen gelernt: Vollkommen mit Sauerftoff geliutigtes K.lisí entbült vermuthlich 6 Proportionen; deun aach der Analjle des Hrn. Chenerix, welche Jurch eise ron Hirn. E. Dary in dem 


\section{$\left[\begin{array}{lll}6 & 65 & 1\end{array}\right]$}

Laboratorio der Royal Inflitution gemachte beffätigt wird, muls überoxygenirt-Lalzfaures Kali beftehn in 100 Theilen aus 40,5 Theilen Kalium, 32,9 Th. oxygenirt- falzfaurem Gas und 45 Theilen Sauerftoff.

Dals bei frerkem Erhitzen von Peroxyd des Kaliums in oxygenirt-falzfaurem Gas aller Sauerftoff ausgetrieben wird, und blofs eine Verbindung von Kalium mit oxygenirt-\{alzfaurem Gas exttheht, hale ich angeführt: Es frhien wir nicht unwahrfcheinlich zu feyn, dafs in mäfsigen Temperaturen eine Verbindung des Peroxyd ait oxygenirter Salzfäure bewirkt werden künne, und ich habe Urfache zu glauben, dafs diefem wirklich fo fey. Ich machte ein Peroxyd durch Erhitzen von Kalium mit ungefahr 2 Theilen Salpeter, und liefo oxygenirt - falzfaures Gas hinzufteigen. Es wurde verfchluckt. Beim Schmelien entwich zwar etwas Sanerftoffgas, doch blieb ein Salz zurück, aus welchem Schwefelfäure fowohl oxygenirt-Lalzfaures als gemeines falz. Caures Gas entband.

In dem Procels, durch welchen man das überoxygenirt - falzfaure Kali elhält, wird offenbar ein 'Theil des Kali's durch feine Verwandtfchaft zum oxygenirt-falzfauren Gas zerfetzt, um falzfaures Kali zu bilden; der Sauerftoff deffelben wird aber nicht frei, londern vereinigt fich, während er im Entbinden begriffen ift, mit einem andern Antheile Kali zu Peroxyd, und diefes mit oxygenirt - falzfaureas Gas. Aus den vorhin berechneten Zahlen lafAunal. d. Phyfik. B. 30. St. 1. J.1811. St.g. E 


\section{$\left[\begin{array}{lll}6 & 66 & ]\end{array}\right]$}

fen fich leicht die Proportionen ableiten, welche zu diefen Veründerungen erfordert werden: Es müffen 5 Proportionen Kali, gleich 240 Grains, zerfetzt rerden, um mit gleich viel Proportionen oxygenirtfalzfaurem Gas, gleich I G4,5 Grains, 5 Proportionen falzfaures Kali, gleich 367 Grains, zu erzeugen; und es vereinigen fich 5 Proportionen Sauerltoff, gleich 37,5 Grains, mit I Kali, gleich 18, und mit I oxygenirt- Talzfaurem Gas, gleich 32,9, zu einer Tripel - Verbindung, um i Proportion über oxygenirtfalz faures Kali, gleich I18,4 Groins, zu geben.

3) Veber dle Verbindungen der Mocalle aus don Eiden mis Sauerfioff und mil oxrgentro-falsfourem'Gas.

Salzfaurer Baryt, Calzfaurer Strontion und falz. faurer Kalk find, wenn man lie lange Zeit in Weirsglïhehitze erhalten hat, durch keine einfache Anziehung zu zerfetzen. So z. B. werden fie durch trockne Boraxfäure nicht verändert; fobald aber Walfer hinzugethan wird, geben fie fogleich SalzFaure und ihre Erclen her. Diefer Umltand briagt mich auf die Vermuthung, dafs diefe drei Salze aus den metallifchen Bafen ihrer Erden, welche ich Barium. Strontium, Calciun genannt habe, und aus oxygenirt-falzfaurem Gas beftehn, und dic Verfache, welche ich darüber habe anftellen küanen, beflätigen diefen Schluls.

Wens man Baryt. Strontion oder Kalk in oxygenirt-falzfaureas Gas bis zum Rochglülta erhitat, fo entftelien gana dielelben Kürper, wio die 


\section{[ 67 ]}

trocknen falztouren' Sake, ind Snuerltof wird aw den Erden ausgetrieben. Ich habe indels nie one Co vollitändige Zerfetsung diefer Erden 'durch oxygenirt-falzfaures Gas zu bewirkon-vermocht, dafs ich Jie Menge des Sauerftcffs, welche in einer gegeteren Menge Erde enthalten if; hatto aumitteln künnen. In drey mit grofser Songfali angeftellten Verliurhen fond ich jedoch, dafs freh s Maats Saverftoffgas entband für je 2 Maots oxygenirt-falzfaures Gas, welche rerfchlackt wurden.

Ueber die Einwirkung der Balen der alkalifchen Erden ouf oxygenirt-falzlaured Gas habe ich noch keine Verfuche angeftelt, zweite aber nicht im geringften, dafs lie fich mit diefpm Ges direct verbinden, und damit trockne falzlaure Salze bilden.

In meinen letzten Verfuchen uber die Metallifirung der Erden durch Amalgamation "), wendete ich rorzügliche Aufmerkfamkeit auf den Zuftand der Producte, welche entitehn, wenn man den Hïckliand der Amalgame der Luft ausfetzt. Ich fand, dafs Baryt, der auf diefem Wege gebildet ift, in heftiger Weifsglühehitze nicht fchmelzt, und dafs fo erzeugter Sirontion und Kalk, wenn fie óeglüht werden, kein Wafter hergeben. Baryt aus Kigfiali len der Erde bereiter. ift, wie Hr. Berthollet gezeigt hat, ein fchmelzbares Hydrat, und ich habe gefunden, dals aus diefer Erde Feuchtigkeit entwich, wenn fio durch oxygenirt-falafaures Gas E 2

-) Annalen N. B. B. VUS. S. $186 . \mathcal{E}$

oilhers. 


\section{$\left[\begin{array}{lll}68 & \text { [ }\end{array}\right.$}

serfet2t warde. Der Kalk in dem Ralk-Hydrace wird von oxygenirt-falz faurem Gas weit Ichnellex als gebrannter Kalk serlegt, indem diefes den SauerItoff delfelben sugleich mit dem Walfer fchnell eustreibt.

Ich exhitate etwas gebranaten Kalk in einer mit falzfaurem Gas gefüllten Retorte; Iogleich entffand Waffer in grofser Menge, und es lafst fich kaum zweifeln, dafs es ron dem Wafferltoff der Salzäure, der mit dem Sauerftoff des Kalks in Verbindung trat, herrïbrte.

Da Kalium das Kochfalz fo fchnell zerfetzt, fo hoffte ich, ee werde auch falzfauren Kalk zerfetzen, und dadurch auf ein leichtes Mittel fuhren, Calcium au erhalten. Die Umfïnde diefes VorSuchs find Sehr ungünftig wegen der Schnolligkeit, womit falefaurer Kalk'Wafer einfchlürft, und weil es fehr Ichwer bält, ihn felblt durch Weifoglühehitze von den letzten Antheilen Wafler zu befreyen. Dennoch erhielt ich, als ich in einer Retorte aus fchwer Ichmelzbarem Glafe Kalium in Berührung mit dem Salze ltark erhitzte, einen dunkelfarbigen, durch eine glaGge Mafle verbreiteten Kürper, der in Waffer ein lebhaftec Aufbraufen erregto. Alles Kalium war verfchwunden, und die Ketorte hatto aine Hitze ausgeftanden, in welcher Kalium gänzlich verfliegt. - Aehnliche Refultate erhiolt ich mit falzfaurem Strontion und mit falzfaurem Baryt, letztere jedoch minder deutlich, da mehr Kalium unverändert überdefuillirte. Entwedor wurden in 


\section{3}

diefen Procetion die Bafen der Erden gans oder zum Theil ton oxygenirt-Galsfaurem Gas befreyt, oder das Kalium war mit den falzfauren Salzen in dreifacho Verbindungen getreten. Ich hoffe bei zpkünftigen Gelegenheiten hierüber ins Reine zu kommen.

Verbindungen von ralzfaurem Gas mit Magna fa. Thonerde und Kiefelerde werden alle durch Hitze zerfetzt; die Szure entweicht und die Frde bleibt frei zurück. Aus diefem Umitande vermuthote ich, oxygenirt-falafaures Gas wiirde aus dieTen Erden den Sauorfioff nicht austreiben; eino Vermuthung, welche die Verfuche beftätigen. Ich erhụtzto jede dor drai Erden in orygenirt-Calzlaurem Gas, bis fie roth glühton, ea ging in ihnen aber keine Veränderung vor.

Die HH. Gay-Luflae and Thenard haben gezeigt, dafi Baryt Sauerlfoffges rerfchlucken, kann, und whrfcheinlich giebt \& Peraxyde der Bafen der Erdea, da zu Folge der Verfuche des Hrn. Chevenix die mehrelten Erden fähig lind, zu überoxygemirt-falsfouren Solzen au worden.

Ich habe verfucht Kalk mit mehr Sauerftoff zu verbinden, indem ich ihn in überoxygenirt - falzfaurem Kali erhitzte, jedoch ohne Erfolg; wenigftens eatwickelte fich aus ihm, als ich ihn nach dielem Procefle mit Wafter verband, kein Sanerftoffges. Der zum Behuf des Bleichens gebildete, fogenannte überoxygenirt-Lalzlaure Kalk lälst, wie ich finde, Sauerftoff in der Hitze fahren, und wird dabai zu Calz Taurem Kalke: 


\section{$\left[\begin{array}{lll}70 & ]\end{array}\right.$}

Aus den Proportionen, welche ich in meiner letzten Baker'Tchen Vorlelung gegeben habe, die aber nach den Analylen fchwefelfourer Salze berechnet find, folgt, dafs, wenn man falzfouren Baryt, falzCauren Strontion und falz fauren Kalk als aus I Proportion oxygenirt-Calzfaurem Gas und I Proportion Metall beftehend betrachtet, fie auf 32,9 Gewichtstheilen oxygenirt-falzfaurem Gas 71 Gewichtstheile Barium "), 46 Strontium und 21 Calcium enthalten müften. Um zu unterfuchen, in wie fern diefe Zahlen genau find, zerfetzte ich von jedem diefer Salzfauren Salze, nachdeau fie zuvor bis zum Weilsglüho erhitzt warden waren, 50 Grain mit falpeterfanrem Silker, und lammelte, wulch, erhitzte und wog den Niederfchlag. Auf diefe Art behandelt, gaben 50 Grain falzlaurer Baryt 68; falzfaurer Strontion 85, und falzfaurer Kalk 125 Grain Hornfilber. Nun erhellt aus den gleich onzufübrenden Verlu. chen, dafo Horthilber aus 12 Theilen Silber auf 3.y Theilen oxygetirt - falz faurem Gas belteht; folglich wird Barium diurch 54,1, Strontium durch 46,8 , und Calcium durch 20,8 dargeftellt.

5) Vober die Verbindungen dor gewöhnllchen Mecalle mire Sauerfoff und mit oxygenirt-falzfaurem Gas.

Es laffen Gch innerhalb der Gränzen, welche' für Vorlefungen diefer Art üblich find, nur die

') Logt man Jamea Thont Cauren Baryts bei der Berecboung zum Gruade, und Cchaizt die Schrefelfaure ouf 36 . To wird Barium ungefibs durch die Zahl 65,5 dargeftelle.

Davy. 


\section{$\left[\begin{array}{ll}71 & ]\end{array}\right.$}

Auffenlinien ron den rielen Verfuchen geben, welche ich über die Verbindungen von oxygenirt - falz Caurem Gas mit den Metallen gemacht habe; ich mufs mich daher anf allgemeine Ueberficliten der Verfahrungsart und der Refultate befchränken.

Ich habe mich in allen fulgenden Verfuchen kleiner Retorten mit grünem Glale bedient, von 3 bis 6 Kubiksoll lnhalt, welche mit Hähnen (Ropcocks) ver(ehn waren. Nachdem der metallifche Kürper in die Retorte hineingebracht war, wurde Ge luftleer gepumpt und mit dem Gas, auf welches er einwirken Collte, gefüllt, dann wurde die Retorte mit einer Weingeiflampe erhitzt, und nach dem Abkiihlen derfelben das Produet anterfucht und das Gas analyfirt.

Alle Metalle, mit welchen ich diefe Verluche angeftellt habe, verbrannten, wenn fie in dem oxygenirt - Calzfauren Gas erhitzt wurden, mit Ausnahme von Silber, Blei, Nickel, Kobalt und Gold. Die flüchtigen verbrannten nit Flamme, und zwar Arjenik, Spie/sglanz, Tellurium und Zink mit einer weifsen, Queckfiber mit einer rothen Flamme. Zinn wurde weils gliihend, Eifon und Kupfer wurden roth glühend, Scheelium und Manganes dunkelroth glühend; auf Platin gefchah in einer Hitze, bei welcher das Glas fchmilzt, faft gar keine Einwirkung.

Folgendes find die Producce, welche bei dem Verbrennen der Metalle in oxygenirt - Lalzlauren Gas entitanden. 


$$
\text { [ }: 2]
$$

Aus Arlenik Arfenikbutcer : eine dichte, helle, höchft Aüchtige Fluilligkeit, welche die Electricität nicht lejtet, kein grofses Ipecif. Gevicht hat, und beim Zerfetzen durch Waffer Arfenik-Oxyl und Salzfäure giebt. - Aus Spiefsglanz Spiefsglanzbutter: ein leicht Ichmelzbarer und Hüchtiger fefter Kürper, von der Farbe des Hornflibers, und von grofser Dichtigkeit, der beim Abkiihlen in fechs. feitigen Tafeln kryftallifirt, und beim Zerfetzen durch Walfer ein weilses Oxyd hergiebt. - Diefem Kürper gleicht in Teinen Gnnlichen Eigen Tchaften das Product aus Tellurium, welches gleichfalls mit Wafler fich in ein weifses Oxyd verwandelt. Auch das Product aus Zink ift demfelben on Farbe ähnlich, doch weniger flüchtig. Das Product aus Queckfilber ift ätzender Sublimat.

Die Verbindung aus oxygenirt - falzlaurem Gas und Eifen ift glänzend braun, von cinem Glanze, der fich dem metallifchen nähert; und fpielt Regenbogenfarben wie die Eilenftufen von Elba. Sie verfliichtigt fich in mälsiger Hitze, und füllt das Gefäls mit fchönen kleinen, ausnehmend glänzenden Kryflallen, die fich in glänzende Tafeln zufanmen häufen, deren Geftalt ich nicht beflimmen konnte. Wenn Waffer darauf einwirkt entfleht, rothes lalzfaures Eifen.

Kupfer bildet ein glänzendes roth-braunes Product, das in kleinerer als der Rothglühehitze fchmelzt, beim Abkühlen kryffallilirt und halb durchficltig wird, und bein Einwirken von Waffer eine 


\section{$\left[\begin{array}{lll}73 & 3\end{array}\right]$}

griine Flüligkeit und einen griinen Niederfohlag hergiebt. ")

Das Product aus Manganes ift in dunkler Roth glühehitze nicht flüchtig. Es ift dankelbraun, wenn aber Waffer darauf einwirkt, wird es heller braun, und in der Auföling bleibt falzfaures Manganes, welches die Lakmulstinktur nicht röthet; der chokoladenbraune Rückftand ift nicht auföslich. ")

Scheelium giebt ein dunkel-orangefarbnes Sublimat, welshes beim Zerfetzen durch Waffer Salzfaure und gelbes Scheelium-Oxyd hergiebt.

O En wire der Mïhe werth su untorfuchen, ob diefer Niederfchlag, der aus oxygenirt-fals-faurean Kupfer durch Waffor gebildet wird, nicht euch viollolcht nicht-gofartigtes fals[enres Kepfor-Hydrüe (a hydraced fabmurtece) wäs, und in Toiner Zuhmmenfotsung mit dem kyffallifirtén falsfauren Kupfer aus Poru übercinftimmte.' Diefes lotstere giebt, wie icb Gode, in der Hitse Sabfinure und Waller. Die von Bojlo. entdockto rofina caprt, wolcho durch Erbitsung von Kupfor mit ütsendem Sublimat enuteht, entbält wabrfehoinlich our I Proportion oxygenirt-fals[aures Ges. indels das oben erwähnte Prodact a Proportiones enthalien mufo.

Devy.

-) Salsfaures Manganes, durch Anföfen von Mlanganes-Oxyet in Salzfiure gebildet,-ift eine neutrale Verbindung, und lifse fich durch Hitso serfecsen, wobei falshaures Gas entweicht, and braunes Manganes-Oxyd surück bleibt. In diefer Hinficht erfchoint Langanes als oin Mittoliting ewiIchen den alien und den neu ontdeckten Metallen. Salzfaures Manganes wird nemlicb gleich der falstaurer Magnefia zerfeut. und das Oxyd doffelben if, fo weit meino Vesfucbe roicber, das einsige onter den lïnger bekannten Metallen, wolches die faure Kraft doe falafnuren Gas neutralifirt, fo dafs diefes in der Aubüfung blaue Planzenfarben nicht vevandere.

Davy. 


\section{[ 74 ]}

Zinn giebt Libav's Fluif/gkeif, welche fich durch Wafler in falzlaures Zinn im Maximum der Oxydirung verwandelt.

Silber and Blei geben Hornfilber und Hornblei, und Wismuth Wismuth-Butter.

Folgendes find die Mengen von oxygenirt-[alzfaurem Gas, welche von a Grains eines jeden Metalls ver(chluckt wurden : *) Von

Arfenik 3,6 K.Z. Zink 3,2 K.Z. Wismuth 1,5 K.Z.

Spie[slgans 3,1 - Eifen 5,8 - Bloi 0,9 -

Tellur $2,4-\operatorname{Zinn} 4 .-$ Silber $\left.0,9-{ }^{-0}\right)$

Queckfilber 1,05 - Kupfer 3,4 -

Bei meinen Verfuchen iiber die Einwirkung des oxygenirt-Lalzfauren Gas au[ Metall-Oxyde, wurden die Oxyde ron Blei, Silber, Zinn, Kupfer, Spiefsglanz, Wismuth und Telluir in einer

") Das orggenirt - Calafauro Gas war in diefen Vorfuchen nicht von Feuchtigkeit befreit worden, und da ich mich mellingner Häbno bediento, kann ein wenig $G a s$ von der Oberfäche des Metalls verfchlucke worden fejn; diefo Procello geben daher blofse Annäherungen an dio wahre Zufammenfetsung der oxygenirt - Calzfauren Metalle. Die Verfuche mit Blei, Tellur, Eifen, Spiefoglanx, Kupfer, Zinn, Queckfliber und Arfenik wurden an drei auf einander folgender' Tagen angeftellt, an welchen der Barometcrftand ewitchen 36,26 und 30,15 engl. Zollen und der Thermometerftand swifchen 63,3 bis 61 'T. variirte. Bei dem Verfuch mit dem Silber ftand das Barometer auf 29,9 engl. Zoll und das Thermometer auf $52^{\circ} \mathrm{P}$. Davy.

-) Das Silber aahm hierbei an Gervicht um o,6 Grain su; ein Refulset, welches fehr gut mit einem Verluche meines Bruders John Davy übereinftimat, in welchem 12 Grain Silber su 15.9 Grsin wurden, indem lio Gich in Hornfil. ber rerwasdeiten.

Davy. 


\section{[ 75$]$}

Hitze, welche das Rothglühen nicht erreichte, zerfetzt, und zwar die Oxyde der flüchtigen fchneller als die der feuerbeftandigen Metalle. Auf Kobalsund Nickel-Oxyd ging die Einwirkung kaum ror fich in der Hitze des dunkelrothen Glïhens. Rothes Eifenoxyd wurde in ftarker Rothglühhitze nicht angegriffen, indefs die Zerfetzung des fchwarzen Eifenoxyds in einer viel niedrigern Temperatur vor fich ging; und Arfenikfäure erlitt keine Verinderung in der gröfsten Hitze, die lich einer Glasretorte gebon liefs, während das weifse Arfa nikosyd fchnell zerfetzt wurde.

In den Fällen, in welchen Sauerftoffgas ausgetrieben wurde, betrug die Menge deffelben genau fo viel, als ron dém Metall war verfchluckt worden. So 2. B. verfchlucken 2 Grains rothes Queckfilber-Oxyd o,9 Kub. Z. oxygenirt - falzfaures Gas, und geben 0,45K. Z. Sauerftoffgas her. Zwei Grain dunkel olivenfarbenes Oxyd aus Calomel, durch Kali abgefchieden, verfchlucken ungefähr o,94 K. Z. oxygenirt - Calz laures Gas, und geben 0,24 Kub. Z. Sauerfloffgas; in beiden Fälen entfteht ätzender Sublimat. ")

-) Icb babe swrei verfchiedene Analyfen von äsendem Subll. mat und vos Calomel mit vieler Sorgfalt gemecht. 100 Grain ätzender Sublimat, die durch 90 Grain Kali-Hydrat zerfecst wurden, gaben mir das erllo Mabl 79,5, das 2weito Mahl 78.7 Grain orangefarbues Queckfilbes-Oxyd, wovon 40 Grain 9,15 K. Z. Sauerltoffgas hergaben; und mit falpeterfaurem Silber eniftenden eus 100 Grain äisen. dem Sublimat das erfte MIabl 102.5. das wweite Mabl 103.4 Grain falzlaures Silber. - Dugegen gabea 200 Grain 


\section{$\left[\begin{array}{ll}76 & ]\end{array}\right.$}

Beim Zerfetzen ron weifsem Zink - Axyd wurde genau halb fo viel Sauerftoffgas, dem Volumen nach, ausgetrieben, als oxygenirt - falzfaures Gas verfchluckt.

Von vorziiglich interellanter Art war die Veränderung, welche bei dem Zerfetzen des fchwarzen Eifenoxyds und des weifsen Arfenik-Oxyds eintrat. In beiden Fällen entwich kein Sauerftoffgas, fondern in dem einen Fall wurden das eifencchü[fige Sublimat und rothes Eilenoxyd, und im andern Falle Arlenikbutter und Arfenik fäure gebildet. Zwei Grains weifses Arfenik - Oxyd verfchluck-

Calomel beim Zerletsen durch 90 Grain Kali-Hydrat das or. Ite Mabl 82, des zweite Mabl '83 Grain ollyenfarbiges Queckfilber-Oxyd, von denen 40 Grain $4,8 \mathrm{~K}$. Z. SauerItoffgas beim Erbitzen entbanden; und as 100 Grain CaJomel bildeten fich das erft Mahl 58,75, das sweite Mabl $57 \frac{x}{2}$ Grain Hornfilber.

Ich ferzo das mehrfte Zutrauen in der swoiten Analyfe; indels geht aw beiden hervor, dals bei einerlei Mengovon Queckfilber ätsendes Sublimat genau noch ein Mabl [o vicl Sauerftoff als Calomel enthält, und dats gleirbfallo boi gloicher Queckfilbermengo in dom orangefarbnen Oxyde noch ein Mabl to viel Sauerifoff als in dem [cbwarzen Quecljilberoxyde enthalten ift. Dio Olivenfarbe den Oxyds aus Calomel rührt von einer kloined Boimengung von orangefarbnem Oxydo her, wolchee lich auf Koften deo Wallers bildet, das sum Niederfcllagen gebraucbt wird. Nimmt man eine kochend beilse Aufölung von Kali, to ift die Farbe des Oxyds ftets lichwarz, fio wird aber, wenn man ein wenig orangefarlunes Oxyd dasu reibt, olirenfarbig. Man hat behauptet, das nliventarbne Oxyd, welches aus Calomel durch Kali . Hydrat niedergefchlagen wird, eathalte Salzraure, [ey aber danit nicht gelätigt; ieb habc indels darin nio eine Spur von Salefiure entdek. 


\section{[ $\begin{array}{ll}77 & 3\end{array}$}

ten 0,8 Kub. Z. oxjgenirt - Falzfaures Gas. ") - Ich aweifle nicht, dafs fich diefelbe Erfcheinung noch in andern Fällon zeigen wird, in welchen das Metall rerbältnifomäfsig nur eine kleine Anziehung für oxygenirt - falzfaures Gas hat, und verfchiedener Grade ron Oxydirung fahig ifl, und in welchen das Peroxyd gebraucht wird.

Ich habe nur einen einzigen Verfuch gemacht, ein gewöhnliches Metalle - Oxyd durch Salz fäure zu zexletzen, nemlich das falbe Zinn-Oxyd; w entftand Walfer und Libav's Flülligkeit.

Betrachtet man die Milchungaverhältmile, welche fich sus dem Volumen des oxygenirt-falzfauren Gas, das die rerfchiedenen Metalle resfchlucken, berechnen laften, in Beziehung auf dip Mengo Sauerftoff, welche fie erfordern, um in Oxyde verwandelt su werden, fo geht aus den Verfuchen, welche ich angefuhrt habe, hervor, dafs in ihnen entweder eine, oder 2wei, oder drei Proportionen

Ken könten, went es gut gewafeben worden war. Bo ift viebt leicht bei den Analyfen der Queckflitber. Oxyde völ. lige Genavigkeit su erreichen, de ihnen Walfer anhängt, welches fich nicht gans austroiben lafert, obno defs etrrae Sauerfoff ontweicht. In jedom meiner Vorfacho lammelto fich in dem Halle der Retorte ein renig Thau, obgleich ich des Oxyd bis übor $912^{\circ} \mathrm{F}$. erhiter hatte. So dafo die 40 Grain ein wenig su hoch gefchäut find. Dery.

-) Das Beftreben des wolfsan Asfonilk - Oxyds su Arfenikfäure su worden, seigte fich bei der Einwirkung delfelben auf gefchmolsnes Reli - Hydrat auf eine aufiallende Weifo: das Watter in dem Hydrate wird fchnell zerfeut, und debei Arfenik-Waitorfoof entbuadea und asfonikfourea Kali ge bidder.

Dary. 


\section{[ 78 ]}

oxygenirt-falzfaures Gas fich mit Einex Proportion Metall verbinden, und daher müfste es.leicht lega, aus der bekannten Zufammenfetzung der falzfauren Salze die Zahlen abzuleiten, welche die Proportionen darftellen, in denen man lich denken kann, dals diefo Metalle in andre Verbindungen eingehn. *)

\section{Allgomeino Schlufsfolgon and Bemerkungen durch Vorfuche erlüutert.}

Alle Folgerungen, welche ich aus den neuen Verfuchen zu ziehn gewagt, die ich in meiner letzten Vorlefung der küniglichen Societät mitgetheilt habe. werden durch die ganze Reihe dieler neuen Unterfuchungen, wie es mir fcheint, beftens beffititigt.

Oxygenirt-falzfaures Gas vereinigt fich mit verbrennlichen Kürpern zu einfachen Zufammenfetzungen aus zwei Beftandtheilen; und wenn es auf Oxyde einwirkt, treibt es entweder den Sauerftoff derfelben aus, oder veranlalst ihn, in neue Verbindungen zu treten. Wollte man behaupten, der Sauerfoff riibre in diefen Fällen von dem oxygenirt-\{alzfauren Gas und nicht von den Oxyden

-) Nach den in der vorvorigen Anmerkung engefubrten Verfuchen muls die Zahl, welche die Proportionen darfellt, in velcbem fich das Queckfalber verbindet, ungefiabr 200 Teyn; und die $Z_{2}$ bl für das Silber aacb S. 74. ungefähr 100. Die Zahlen für die endern Metalle laflen fich aus den auf derfelben Seite befindlichen Angaben ablciten, welche indels, nach dem was ich angefühit habe, keine grofse Ge. nauigkeit geben künnen.

Davy. 


\section{$\left[\begin{array}{ll}79 & 1\end{array}\right]$}

her, fo lälst fich fragen, warum die Menge deflelben immer gonau der in dem Oxyde enthaltenen Menge gleich ift, und warum fie in einigen Fällen, wie denen mit Kalium- und Natronium - Oxyd im Maximo, in keinem Verhältnilte mit der Gasmenge fteht?

Enthielte das oxygenirt-Talzfaure Gas irgend eine mit Sauerftoff verbundne Säure, lo miifste fich diefe in der fülligen Verbindung zeigen, welche aus I Proportion Phosphor und a Proportionen oxygenirt a falzfaurem Gas zufammengefetzt ift; denn unter diefer Vorausletzung müfste diefe Fliifligkeit aus Salzfäure (nach der alten Hypothele, frei vor Waller) und aus Phouphor(ïure beftehn; diefe Verbindung hat aber keine Wirkung auf Lackmuspapier, und wirkt unter den gewöhnlichen Umftänden nicht anf die lixen alkalifchen Bafen, 2. B. nicht auf trocknen Kalk oder trockno Magnefia. Oxygenirt-falzfaures Gas mufs fich, gleich dem Sauerfoff $g$ as , in grolser Menge mit befondern verbrennlichen Körpern verbinden um eine Säure zu bilden; an Wafferftoff gebunden, röthet es.das trockeafte Lackmuspapier angenblicklich, wenn gleich die Verbindung ein Gas ift. Dex Natur der Säuren entgegen treibt es Sauerftoff aus Oxyden im Minimo aus, und verbindet fich mit Oxyden im Maximo.

Wird Kalium in axygenirt-falzfaurem Gas verbranint, lo erhält man eine trockne [wafferfreye] Verbiadung. Nimmt man mit Sauerftoff verbun- 


\section{$\left[\begin{array}{lll}80 & ]\end{array}\right.$}

denes Kalinm, fo wird aller Sauerfoff ausgetrieben, und diefelbe Verbindung gebildet. Es würde gogen die Logik leyn, behaupten zu wollen, dols diele genaue Menge ron Sauerfoff von einem Körper aufgegeben werde, ron dem man nicht wille, dafs er zufammengefetzt ift, während wir von der Exiftenz derfelben in einem andern gewils find und alle Fälle laufen einander parallel.

Dafs Sauerftoff in dem oxygenirt-falzfauren Gas vorhanden fey, find vielleicht einige aus dem Umftande zu fchliefsen geneigt, weil es durch Einwirkang von Salzfäure auf Oxyde im Maximo, oder auf überoxygenirt-falzfaures Kali gebildet wird. Bei einigem Nachdenken zeigt fich indefs bald, dafs die Erfcheinungen bei diefen Einwirkungen mit meinen Anfichten völlig übereinltimmen. Wenn man falzfaures Gas über trocknes Manganes-Oxyd in Maximo erhitzt, fo wird, wie ich finde, fehr schnell Wafler gebildet und oxygenirt-falzfaures Gas hervorgebracht, das Peroxyd aber wird braun. Jetzt, da wir wiffen, dals falzfaures Gas aus oxygenirt-falzfaurem Gas und Wafferftoff befreht, giebe es keine einfachere Erklärung des Erfolgs, als die, dars der Wafferftoff der Salzläure fich mit Sauerftoff des Peroxyds zu Waffer verbinde.

Scheele erklärte die bleichende Krafc des oxygenirt-falzfauren Gas daraus, dafs er annahm, es zerftöre die Farbe, indem es fich mit dem Phlogifton derfeiben verbinde; Berthollet fetzt die bleichende Wirkung darin, dafs diefes Gas den Farben 


\section{[ $8 \mathrm{x}$ ]}

Sauerftoff zufïhre. Ich habe einen Verfuch gemächt, welcher $2 u$ beweifen fcheint, dafs das reine Gas unfähig ift; die Planzeáfarben zu verändern, und dafs ihr Vermögen zu bleichen lediglich darauf beruht, dals fie die Eigenfchaft hat, das Waller zu zerfetzen, und den Sauerftoff deffelben frei zu machen. - Ich füllte nemlich einen Glasballon, der trocknen, gepulverten, falzfauren Kalk enthielt, wit oxygenirt-falzfaurem Gas, und that in einen andern Ballon, worin fich ebenfalls trockner falzfaurer Kalk befand, exwas trocknes mit Lackmus getärbtes Papier, nachdem es zuvor erhitzt worden war. Nach einiger Zeit pumpte ich diefen Ballon luftleer, und fetzte ihn mit dem erften in freye Verbindung, während das Lackmuspapier vermöge des dazu eingerichteten Habns der Wirkung des einftrömenden Gas ausgeletit war. Es zeigte fich keine Farbenänderung, und noch nach zwei Tagen war kaum irgend eine Veränderung der Farbe bemerkbar.

Ein ähnliches getrocknetes Lackmuspapier, da ich in oxygenirt-falzfaures Gas brachte, worauf kein falzfaurer Kalk eingewirkt hatte, wurde augenblicklich weifs *). Lackmuspapier, das man nicht zuvor getrocknet hat, erleidet in trocknem

-) Diefe leztern Verfuche habe ich in dein Laboratorio der Dablin Soctely. (die mehrften der rorhorgehenden in dem 'Laboratorio der Rayal Inftucuton) angatellt, und auf lie mich zu beziehn, haben die Vorfteher (Managers) diefer nützlichen üfendichen Anftatt mir orlaube. $D \in v y$.

A.rnal.d. Phylik. D. 39. St. r. J. 181?. St. g. 


\section{[ $\begin{array}{ll}82 & ]\end{array}$}

oxygenirt-falz (aurem Gas diefelbe Veränderung, nur langfamer.

Die überoxygenirt - falzfauren Verbindungen fcheinen ihre bleichende Kraft allein ihrem locker gebundnem Sauerftoff zu verdanken; die gewöhnlichen Metalle haben ein ftarkes Beltreben, einfache Verbindungen mit oxygenirt - falz faurem Gas einzugehn, und der Sauerfoff wird hierbei aus ihnen leicht ausgetrieben und angezogen.

Man findet in allen chemifchen Schriften angegeben, das oxygenirt - falzfaure Gas condenfsre fich in niederen Temperaturen und kryftallifire. Ich babe mich durch einige Verfuche überzeugt, dals diefes nicht der Fall if. Aufüfungen von oxygenirt - lalzfaurem Gas in Waffer frieren eher als reines Waffer; aber das reine, durch falzfauren Kalk getrocknete Gas leidet in Temperaturen von $-40^{\circ}$ F. keine Veränderung irgend einer Art. Der lrrthum fcheint daher zu riibren, dafs man oxygenirtfalzfaures Gas in Flafchen, welche Feuchtigkeit enthielten; der Kälte ausgefetzt hat.

Ich habe verfucht, Boraxfaure und Phosphorfäure durch oxygenirt - falzfaures Gas zu zerfetzen. jedoch ohne Erfolg; woraus zu erhellen lcheint, dals Boracium und Phosphor eine grofsere Verwandtrchaft zum Sauerftoffe als zu dem oxsgenirtfalzlauren $G$ as haben. Und hierin ftimmen, den oben angegebenen Verfuchen zu Folge, Eifen und Arfenik, walıfcheinlich auch einige andere Mi:talle, mit ihnen überein. - Eine geringere Ver- 


\section{[ 83 ]}

wanduchaft zu dem Sauerftoff als zu dem oxygenirtCalzfauren Gas haben: Kalium, Natroniusn, Cal cium, Strontium, Barium, Zink, Queckfulber, Zinn, Blei und wahrfcheinlich such Silber, Spicjsglanz und Gold.

Ich habe bis jetzt nur fehr wenig Verfuche iiber die Verbindungen der oxygenirt - Calz fauren Kürper unter einander oder mit den Oxyden anftellen künnen. Arfenikbutter und Libav's Fliifligkeit zulaınmengemilcht, erhitzen lich mit einander; die Phosphor- und die Schwefel - haltigen Flïlligkeiten vereinigen fich beide unter einander und mit Libav's Fliiligkeit ohne irgend eine merkwürdige ErScheinung.

Ich hatte in einer grünen Glasrühare Kalk mäfsig erwärmt und trieb das Phosphor-Sublimat (den gefattigten oxygenirt - falzfauren Phosphor) in Dämpfen hiodurch; die Einwirkung war beftig, unter Entbindung von Wärme und Licht, und es entfiand eine graue gelchmolzne MafTe, welche, als ich Waffer zufetzte, falzfauren und phosphorfauren Kalk hergab. - Etwas Dampf des erhitzten Phosphor-Sublimats liefs ich in eine luftleer gepumpte Retorte Ateigen, worin fich getrocknetes Lackmuspapier befand; die Farbe deffelben verwandelte Gich langlam in blasroth. Diefe Thatlache licheint der Meinung günltig, dals diefe Subftanz eine Sicure fey; da aber ein wenig Wafferdampf in der Hetorte gewefen feyn künnte, fo ifl der Verfuch nicht entlcheidend. Auch die ftarke Verwandt$F_{3}$ 


\section{[ $\quad 84$ ]}

fchaft derfelben zum Ammoniak lipricht vielleicht für diefe Meinung; zwar bilden alle oxygenirt-falzfaure Körper, die ich verlucht habe, mit dem Ammoniak dreifache Verbindungen, aber aus den iibrigen Verbindungen von oxygenirt-Falzfaurem Gas und Phosphor mit Ammoniak wird Plosphor durch eiue mälsige Wärme ausgetrieben, und die zurückbleibende Verbindung ift das Phosphor-Sublimat.

5) Eintge Betrachimngen ilber die Nomenclotur der oxygenirt - Jalyfauren Zufammenfecexngen.

Einen Körper, von dem es nicht bekannt ift, dal's' er Sauerftoff enthält "), und der keine Salz-

-) Wäbrend ich diefen Bogen für den Druck corrigire, finde ich in dem neaften Stück von Nicholfon's Journal. welches am 1 . Februer 18 is erfchienen if, einen fcharfCinnigen Auttats des Hrn. Murray in Edinburg, in welchem bewiefen werden [oll, dafo oxygenirt-falzfaures Gas Sauerftoff enthalte. Hr. Murray detonirt oxygenirt. Falzon Tauree $G_{a n}$ in Uebermaafs mit einer Mengung von WafferAtoffgas und gasfömigem Kohlenftoff- Oxyd, woraus, feiner Vorawsfocsung nacb, koblenfaures Gas entftebt; und vermifcht oxygenirt-falsfaures Gas in Uebermaalo mit Schwefel-Wafferftoffgas, wodurch, feinor Vorampolsung nach, Schwefelfaure oder fchwellige Säure gebildet wird.

In einigen Verfuchen, bei welchen mein Bruder Johg Davy mit mir gearbeitet bat, und die wir iiber augakoch. tem Queckfilber anftellten, habo ich gefunden, dafo, wenn 7 Manfo WaJjorftoffgar. 8 Maals gasfürmiges KohlenstoffOxyd und 20 Mraafo oxysentre - Jalzfautres $G a s$ durch den electrifelien Funken detonirt werden, Ge Gich bis auf ungefahr 30 Mlaafo vermindern, und dafs Calomel an den Seiten der Rübre fich abletza. Nachdem ich trocknes Ammoniak in Uebermaafs, zugefetzt, und Waffer zu dem Rïckftande gebraclut hatte, blieben mebr als 9 Masfs Gas zurück, und diefes war gasförmiges Kohlenfloff-Oxyd, nicht 


\section{$\left[\begin{array}{lll} & 35 & \end{array}\right]$}

[äure enthalten kann, oxygenirte Salyfäure zu nennen, if gegen alle Regeln derjeaigen Nomen-

unreiner, als fich nach der den verfehiedenen Gasarten beigemengteu atmolphärilcben $L$ Luft und dem aus dem Ammonink ausgetriebenen Stickgas vermuthen liefa. Der Sanerftoff in Hra. Murray' kohlenfenurem Gas fclieint daher aus dem Waffer oder aus dem gaförmigen Kohlenftoff. Oxyd hergerührt zu haben.

Schwof ol - WajJorftoffgas, das ich einem Uebermantse von oxygentrt-falzfaurem Gas über trocknem Queckfilbèr zufeizto, entzündete fich in zmei oder drei Verfuchen, und of eniftand falsfrures Gas, welchee Dimpfe des oxygenirt-Talsfaurea Schrrefels enthielt, und mit Ammaniak neutralilirt, falz aures Ammoniak und eire Verbindung von Ammoniak mit oxygenirt - [aly zaurem Schwefel gab. Stieg eize folcbe Mengung Schwefel-Wafferftoffgas und oxygenirt - falzasures Gas in Uebermaafs in die Atmofplüre, fo verbreitete fich ein Geruch nach oxygenirt-falxfaurent Schwefol, und es seigte fich nicht die kleinfte Spur ron Schwefelfiuire oder Ichwefliger Säure. Hätte Mr. M u rray bei der Analýto feiner Rückftände Ammoniak ftatr Waffer genommen, fo würde er, glaube ich, nicht gefchloffen haben, oxygenirt - fals[aures Gis lafto fich mittelft Fulcher Methodon zerfetsen.

Das Detail oiniger endrer Verfuche, wolche wir über diefen Gegenftand angeftellt haben, berühre ich hier nicht; mein Bruder wird lio in einer Antwort auf Herm Mur$x$ ay's Auffatz bekanat machen. Zum Schlulle bemerke ich noch, dafs diefer [charffinnige Chomiker meine Anfichter miforerftanden bat, wenn er lie fur Hypothelen hïlt. Ich lage blos aus, was ich gefehn und was ich gefunden babe. Ea ift móglich, dafe das oxygenirt-falzfaure Gas Sauerftoff enthät, ich kann eo aber darin nicht finden. Ich habe Hrn. Murray's Verfuche mil vielem In. rerefto wiederholt; wenn WaJer bei denfelben aufeer Spiel bleibt, fo werden durch die Refultate derfelben alle meine Ideen über diefen Gegenflend beftätigt, und die hypothetifclien Ideen, welche er mil fo vielem Eiler zu vrr theidigen gefucht hat, erhalten ditrch lie keine Stritze. 


\section{[ 86 ]}

clatur, in welcher nian ihm dielen Namen gegeben hat, und eine Veränderung diefer Benennung fcheint nothwendig zu werden, um den Fortgang der Erürterungen $2 u$ erleichtern, und um richtige Ideen über diefen Gegenftand zu verbreiten. Hätte der grolse Entdecker diefes Kürpers *) ihn mit irgend einem einfachen Namen bezeichnet, fo wiirde es fchicklich feyn, diefen wieder hervorzuziehn; aber dephlogififirce Kochfalzfoure ift eine Benennung, die für den jetzigen Zufand der Wifenfchaften fich nicht mehr eignnet.

Ich bin mit einigen der vorziiglichften chemifchen Naturforlcher zu Rathe gegangen; es fchien uns am fchicklichften $z u$ feyn, einen Namen in Vorfchlag zu bringen, der von einer der bekannteflen und am wehriten charakteriltifchen Eigenlchatten des oxygenirt-falzfauren Gas, run der Farbe defrelben, endehnt ift, nemlich Chlorine oder Chloric Gas *); ein Name, den man auch dann unverän. dert würde beibehalten künnen, wenn man künttion finden follte, Jafs diefer Kürper zulammengeletzt fey, ja felbft, dals er Sauerfoff enthielte.

Von den mehrften Salzen, welche man bis jetzt für falzfaure gehalten hat, wiffen wir nicht, dals Sse irgend einen Antheil Salz äure oder irgend einea Antheil Sauerftoff enthalten. So findet fich in Libav's Fliilligkeit, wenu gleich W'affer fie in eine Calzfaure Verbindung verwandelt, allein Zinn und

-) Scheele.

G.

") Von x xupos (grünlich - gelb]. 


\section{$\left[\begin{array}{ll}4 & 87\end{array}\right]$}

oxygenirt - falzfaures Gas, und Hornfilber fcheint unfähig zu feyn, in ein wahres falzfaures Salz verwandelt zu werden. Ich wage es, für dic Verbindungen des oxygenirt - falzfauren Gas mit verbrennlichen Körpern folgende Kenennung vorzulchlagen : „,den Namen ihrer Bafis mit der Endfylbe ane." Auf diefe Weife würde Argentane Hornfilber, Stannane Libav's Flülligkeit, Antimanane Spiefsglanzbutter, Sulphurane Thom[on's [chwefelhaltige Flüffigkeit, Phosphorane das Phosphor-Sublimat, und fo ferner, bedeuten *).

Bei diefer-Benennung lielse fich zugleich die Klafle, su welcher der Körper gehört, und die Befchaffenheit deffelben ausdrücken. Enthielte die Verbindung auf I Proportion oxygenirt-lalzfaurem

-) Für die deutiche chemifcho Spracho find diefo Namen nicht brauchbar. Statt ibrer wüfte ich koine lchicklichrren in Vorfchleg su bringen, als Zufammenfetzungen ains dem Worte Chloran und der deutfchen Benennung der Bafis, z. B. Chloran-Stlber, Chloran-Zinn, ChloranSplefsglanz, Chloran-Schwefel, Chloran-Phoephor u. f. f. Und zwar [chlage ich wit Ablicht die Biegung Chloran (nicht Chlortne) vor, um einestheile durch Aehnlich. keit des Klangs einer Endfylbe an Davy'. Nomenclatur zu erinpern, enderntheils durch die Vorwandlung Chlorine in Chloran darauf binzudeuten, dafo diefes Verbindungen von einer aodern Klafle find, alo dis Verbindungen der verbrennlichen Körper unter einander, welche ich durch Zufammenftellung der Namen diefer Körper be. zeichne, z. B. Schwefel-Zinn, Pbosphor-Blei, u. f. w.; eine Bezeichnung, worin alle deutfche Chemiker den Annalen gefolgt su fevn fcheinen. Wenn. Hr. Dary fich in feinen zukünftigen Arbeiten der von ihm hier vorge[chla. genen Nomenclatur bedienen folltc. fo werde icls fie aut diefe Art im Deutfchen wirdergeben.

Gillert. 


\section{[ 888 ]}

Gas mehrere Proportionen des verbrennlichen Körpers, fo könnte man z. B. dem Namen die Vocale vorfetzen, wobei a zwei, e drei, $i$ vier Proportionen bezeichnen würde; wären dagegen mehrere Proportionen prygenirt-falzfaures Gas mit I Proportion des verbrennlichen Kürpers verbunden, fo mürste man die Vocale dem Namen anhängen.

Salzfäure ift eine Benennụg, die man für die Verbindung des Wafferftoffs mit oxygenirt-falzlaurem Gas beibehalten muls; eben fo die bisherige ,Weife, die Zufammenfetzungen aus diefem Körper und aus den Oxyden nach Art der andern Neutralfalze zu bezeichnen "). So alfo find falzfaures

") Wollte mas den Namen det Salz[äure, aetde murlasique (muriacic acid) in den aclde murtacigue hydrogénd oder actde hydromurtalique (hydrogenased martatic or hydromurtastc acld) verwandeln, und dio diofo Säure entbaltenden Salso murlates hydrogónds oder hydromuria. ces (hydrogenased martates or hydromurlases) nennen, fo mürde es niche lchwer foyn, eine Modification der jetrigen Benennung des oxygenirt - falefouren Gas aufsufinden, welche den neuen Anfichten ontlpräche, und zuglcich ihro Besiebung. zur Salzfaure auidrückte, 2. B. gas demurtastque (demurtaste gas) oder gas oxymuric (oxymurtc gas). und dann wären die Verbindungen aus oxygenirt-falshaurem Gas demuriaces odor oxymurtates zu nennen. Diefes würde ober die Verbreitung richriger Anfichten in diefem Theile unferer chemilchen Renntniffe viel verwickelter und Tchwierigor macken, als die Methodo der Beseichnuig, welcho ich rorgelchlagen habe. Bodenken wir den Zuftend der Kindheit, worin Gich diefo Unterfuchungen befinden, fo dürfte en überbaupt gerathen Seyo, die Annahme irgend einer neuen Benennung für folche Verbindungen furs Erfto noch ausufetzen. Es ift möglich, dafe das oxjgenirt-Galzfauro Gas ein zulammen. 


\section{[ 89 ]}

Ammoniak, falzfaure Magnefia u. ᄃ. f. auch in der neuen Anficht diefer Gegenftände vollkommen richtige Ausdriic'ie.

Dqch ich verweile mich hierbei nicht lïnger. Was ich hier gebe, find blofse Vorfchläge, durch die ich die Aufmerkfamkeit der Naturforfcher auf diefen Gegenftand zu lenken wünfche. Indem die Chemie allmählig immer mehr vervollkommnet werden wird, mülfen manche andere Verände. ruogen in der chemifchen Sprache nothwendig werden. Es ift $z u$ wünfchen und $z u$ hoffen, dafs man fich bei diefen Veränderungen jedesmahl von allen Ipeculativen Anfichten unabhängig erhalten, und die neuen Namen nicht aus der Theorie fchöpfen, fondern vielmehr von irgend einer einfachen und unveränderlichen Eigen[chaft der zu bezeichnenden Körper ableiten werde, und dafs zugleich die Chemiker dahin übereinkommen werden, Gich lediglich willkührlicher Bezeichnungen zu bedienen, um die Klalfe anziudeuten, zu welcher zulamınengefetzte oder einfache Körper gehüren.

gefetater Körper fey, und dafs ee mit dem Sauerftoff ein gemeinfames Element habo; bio jest aber haben wir nicbt mehr Recbt auszulagen, das oxygenirt-Lulzlaure Gas enthalte Sauerfoff, als su behaupten, Zinn enthalte Wafferftoff; Namen follen aber Sachen und nicht Meinungen ausdrücken, und beror nicht ein Kürper zerfetzt ift, mü؟fen wir ihn für einfach nebmen.

Dany. 